\title{
Características clínicas e histológicas de las enfermedades parenquimatosas renales en una muestra de biopsias renales obtenidas entre el año 2002 y el 2017 en el departamento de Caldas, Colombia
}

\section{Clinical and Histologic Characteristic of Renal Parenchymal Diseases in a Renal Biopsy Sample since 2002 to 2017 in Caldas - Colombia}

\author{
๑Héctor Guillermo García Herrera ${ }^{1},{ }^{\oplus}$ César Augusto Restrepo Valencia ${ }^{2, *},{ }^{\bullet}$ Carlos Alberto Buitrago Villa ${ }^{3}$ \\ ${ }^{1}$ Departamento de Medicina Interna, Facultad de Medicina, Universidad de Caldas, Colombia \\ ${ }^{1,2}$ Departamento Clinico, Facultad Ciencias para la Salud Universidad de Caldas, Facultad de Medicina, Universidad de Caldas, Colombia \\ ${ }^{3}$ Unidad renal RTS, sucursal Caldas, Caldas, Colombia
}

\section{Resumen}

Antecedentes: los síndromes renales son manifestaciones clínicas y de laboratorio que indican alteraciones funcionales y morfológicas. La biopsia renal es fundamental en el diagnóstico de enfermedades renales parenquimales y aporta información valiosa sobre la incidencia, distribución y posible control de la enfermedad.

Objetivo: describir las características clínicas e histológicas de las enfermedades del parénquima renal en una muestra de biopsias renales. Métodos: se incluyeron 269 pacientes mayores de 14 años, a quienes se les realizó biopsia renal por cualquier método. Se clasificaron por indicación de biopsia y por tipo de lesión renal primaria o secundaria.

Resultados: el promedio de edad fue de 57,04 (DE $\pm 17,17$ años). La mediana de creatinina fue 1,51 mg/dl (RIC=1,22 - 2,01); y la de TFG por CKD-EPI, de 42,7 mil/minuto (RIC= 30,6 - 56,5). Las indicaciones de biopsia renal más frecuentes fueron enfermedad renal crónica sin causa clara $(46,8 \%)$, proteinuria no nefrótica $(20,1 \%)$, síndrome nefrítico $(8,2 \%)$, lesión renal aguda $(7,1 \%)$, hematuria glomerular con cambio en el patrón $(7,1 \%)$, síndrome nefrótico $(6,7 \%)$ y tasa de filtración glomerular estimada baja para la edad sin causa clara $(4,1$ \%). Los hallazgos encontrados fueron: nefropatía por IgA (20,9\%), nefropatía hipertensiva (19\%), glomeruloesclerosis focal y segmentaria $(11,6 \%)$, nefritis tubulointersticial $(9,7 \%)$, glomerulopatía diabética $(8,6 \%)$, glomerulonefritis membranoproliferativa $(3,7 \%)$ y glomerulonefritis proliferativa extracapilar $(3,4 \%)$.

Conclusiones: la nefropatía por IgA y la glomeruloesclerosis focal y segmentaria son las principales glomerulopatías primarias. La nefropatía hipertensiva y la nefritis tubulointersticial son las principales etiologías secundarias.

Palabras clave: enfermedades renales, glomerulonefritis por IGA, epidemiología, proteinuria, hematuria.

doi: http://dx.doi.org/10.22265/acnef.0.0.300

\begin{abstract}
Background: Renal syndromes are clinical and laboratory manifestations that indicate functional and morphological alterations. Renal biopsy is essential in the diagnosis of kidney parenchymal diseases and provides valuable information in incidence, distribution and possible control of the disease.

Objective: To describe the clinical and histological characteristics of renal parenchymal diseases in a sample of renal biopsies.

Methods: We included 269 patients older than 14 years who underwent renal biopsy by any method. They were classified by indication of biopsy and by type of primary or secondary kidney injury.

Results: The average age was 57,04 ( $\mathrm{SD} \pm 17,17$ years). The median creatinine was $1,51 \mathrm{mg} / \mathrm{dL}(\mathrm{RIC}=1,22-2,01)$ and the GFR for CKDEPI was 42,7 mil/minute (RIC=30,6 - 56,5). The most frequent renal biopsy indications were unexplained chronic kidney disease $(46,8$ $\%)$, non-nephrotic proteinuria $(20,1 \%)$, nephritic syndrome $(8,2 \%)$, acute kidney injury $(7,1 \%)$, glomerular hematuria with change in the pattern $(7,1 \%)$, nephrotic syndrome $(6,7 \%)$ and unexplained low glomerular filtration for age $(4,1 \%)$. The most frequent finding were IgA nephropathy (20,9\%), hypertensive nephropathy (19\%), focal and segmental glomerulosclerosis (11,6\%), tubulointerstitial nephritis $(9,7$ $\%)$, diabetic glomerulopathy $(8,6 \%)$, membranoproliferative glomerulonephritis $(3,7 \%)$, extracapillary proliferative glomerulonephritis $(3,4 \%)$.

Conclusions: IgA nephropathy and focal segmental glomerulosclerosis are the main primary glomerulopathies. Hypertensive nephropathy and tubulointerstitial nephritis are the main secondary etiologies.
\end{abstract}

Key words: Kidney diseases, glomerulonephritis, IGA, epidemiology, proteinuria, hematuria.

doi: http://dx.doi.org/10.22265/acnef.0.0.300

Citación: García-Herrera HG, Restrepo-Valencia CA, Buitrago Villa CA. Características clínicas e histológicas de las enfermedades parenquimatosas renales en una muestra de biopsias renales obtenidas entre el año 2002 y el 2017 en el departamento de Caldas, Colombia. Rev. Colomb. Nefrol. 2018; 5(2):107-117. doi: http://dx.doi.org/10.22265/ $\underline{\text { acnef. } 0.0 .300}$

*Correspondencia: *César Augusto Restrepo Valencia: caugustorv@gmail.com

Recibido: $21.02 .18 \cdot$ Aceptado: 08.06.18 • Publicado en línea: 09.08.18 


\section{Introducción}

L os síndromes renales son manifestaciones clínicas y de laboratorio que indican alteraciones en la integridad funcional y morfológica renal. Para lograr adecuadamente el diagnóstico de las lesiones renales, el segundo paso después del enfoque sindrómico es proceder con una biopsia renal para hacer un diagnóstico

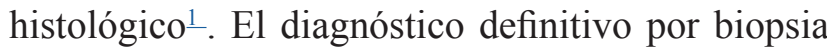
de la patología que afecta a un paciente difiere del diagnóstico clínico hasta en un tercio de los casos $^{2}$. Por lo anterior, la biopsia renal es clave en el diagnóstico final de enfermedades renales ${ }^{\underline{3}}$ y orienta el mecanismo patogénico y, probablemente, la etiología ${ }^{-1}$.

Hasta un $70 \%$ de las causas de enfermedad renal crónica se asocian con hipertensión arterial y diabetes mellitus. El porcentaje restante incluye otras enfermedades renales intrínsecas que típicamente involucran los compartimientos glomerulares o tubulointersticiales y que pueden ser potencialmente reversibles. Identificar las lesiones tratables requiere la realización de una biopsia renal ${ }^{4}$. Por lo tanto, el resultado histopatológico altera la conducta médica en cerca del $40 \%$ de todos los pacientes (enfermedades reversibles) ${ }^{5}$.

Los estudios epidemiológicos con base en la biopsia renal son la mejor forma de evaluar las enfermedades renales ${ }^{3}$. Estas últimas, cuando son confirmadas por biopsia, aportan información valiosa sobre la incidencia, distribución y posible control de la enfermedad con tratamiento dirigido y efectivo.

Sin embargo, la epidemiologia mundial es variable en la histopatología, aun entre regiones, por diferencias en la raza y aspectos socioeconómicos ${ }^{6}$. En Latinoamérica y Colombia, hay pocos estudios sobre enfermedades renales que aclaren el perfil epidemiológico de la región.

\section{Materiales y métodos}

Se revisaron un total de 365 reportes de histología de pacientes, a quienes se les practicó biopsia renal, atendidos por los investigadores en el programa de consulta externa de la unidad renal RTS en el estudio de enfermedades parenquimatosas renales desde 2002 hasta 2017 en la ciudad de Manizales, Caldas, Colombia.

Se incluyeron pacientes mayores de 14 años, a quienes se les hizo biopsia renal por cualquier método y que tenían realización de microscopia de luz, inmunofluorescencia y microscopia electrónica en el procesamiento de la muestra. Se excluyeron los reportes de biopsia de pacientes trasplantados, nefropatía lúpica, estudio de masas o quistes renales, muestra menor o igual a 6 glomérulos o reporte con material insuficiente por el patólogo, reportes repetidos o historia clínica insuficiente o no disponible.

La muestra de la población se tomó por conveniencia, de acuerdo con el número total de biopsias obtenido. Se identificaron las historias clínicas respectivas de los pacientes para extraer los datos relacionados con el sexo, edad, procedencia, valores de creatinina sérica, proteinuria por recolección en 24 horas o por uroanálisis, hallazgos del sedimento urinario y reportes de imágenes renales (ecografía, tomografía o resonancia magnética), realizados antes de la biopsia renal. Se calculó la tasa de filtración glomerular (TFG) por la fórmula Chronic Kidney Disease Epidemiology Collaboration (CKD-EPI) ${ }^{7}$ y se determinó la tasa de filtración glomerular esperada para la edad ${ }^{1}$.

De acuerdo con los datos clínicos y de laboratorio que indicó la biopsia renal, se clasificaron los pacientes en 7 síndromes renales: 1) síndrome nefrótico (proteinuria mayor a 3,5 gramos/24 horas $/ 1,73 \mathrm{~m}^{2}$ de superficie corporal $\left.{ }^{1,8}\right), 2$ ) proteinuria no nefrótica (proteinuria entre 0,3 y 3,49 gramos $/ 24$ horas/1,73 $\mathrm{m}^{2}$ de superficie corporal $\left.{ }^{1.8}\right), 3$ ) síndrome nefrítico (hematuria de más de 3 glóbulos rojos por campo de alto poder en sedimento urinario en 2 muestras separadas por 1 semana, hipertensión no controlada, oliguria, edemas y TFG reducida ${ }^{1.8}$ ), 4) lesión renal aguda (LRA) sin mejoría a las 8 semanas, y con ecografía renal normal ${ }^{1,8}$ (reducción en la TFG por aumento en la creatinina basal de 1 a 1,5 veces o reducción en el gasto urinario - menor a $0,5 \mathrm{cc} /$

108 Características clínicas e histológicas de las enfermedades parenquimatosas renales en una muestra de biopsias renales obtenidas entre el año 2002 y el 2017 en el departamento de Caldas, Colombia 


\section{http://dx.doi.org/10.22265/acnef.0.0.300}

$\mathrm{Kg} /$ hora-), 5) hematuria glomerular con cambio en el patrón (hematuria de más de 3 glóbulos rojos crenados en sedimento de examen de orina más aparición de proteinuria mayor a $300 \mathrm{mg} / 24$ horas $\mathrm{y} / \mathrm{o}$, aumento de azoados $\mathrm{y} / \mathrm{o}$, hipertensión arterial $\left.{ }^{1.8}\right)$, 6) enfermedad renal crónica (ERC) sin causa clara (TFG menor a $60 \mathrm{mil} /$ minuto/1,73 $\mathrm{m}^{2}$ o elevación de la creatinina basal mayor de 1,5 veces por más de 3 meses $\frac{1,8}{}$ ), acompañada de ecografía renal, en la cual se evidenciaran riñones de aspecto normal; y finalmente, 7) tasa de filtración glomerular baja sin causa clara, y menor a la esperada para la edad o menor a $60 \mathrm{mil} / \mathrm{minuto} / 1,73 \mathrm{~m}^{2}$ o elevación de la creatinina basal mayor de 1,5 veces por menos de 3 meses $^{1}$ ). De acuerdo con el reporte de la biopsia, se clasificaron los pacientes por tipo de enfermedad renal parenquimatosa y por etiología primaria $\mathrm{y}$ secundaria.

La recolección de la información se hizo mediante una base de datos diseñada en Google Docs, que fue diligenciada por los investigadores y se procesó por el programa estadístico SPSS 15.0.

Para el análisis estadístico, se utilizaron valores de frecuencia relativa y absoluta con las variables cualitativas. Con las variables cuantitativas, se determinó el tipo de distribución normal o anormal y se usaron medidas de tendencia central y de dispersión, respectivamente (media y desviación estándar - DE-, o mediana y rango intercuartílico - RIC-).

El proyecto fue aprobado por el comité docente de la Universidad de Caldas y por el comité de investigaciones de RTS Colombia.

\section{Resultados}

Se revisaron 365 reportes de biopsia renal. 350 cumplieron los criterios de inclusión. Se excluyeron 37 reportes repetidos, 30 con historia clínica insuficiente o no disponible, 13 con muestra insuficiente o con menos de 6 glomérulos y 1 de paciente trasplantado. La muestra final a analizar fue de 269 reportes histológicos.

El promedio de edad fue de 57,04 años (DE \pm
17,17). 38,28 \% (103) de los pacientes tenía edad mayor o igual a 65 años. 53,5\% (144) eran hombres. $69,14 \%$ (186) eran procedentes de Manizales, 5,95 \% (16) de Chinchiná, 4,09 \% (11) de Villamaría, 3,72\% (10) de municipios fuera de Caldas, 2,6\% (7) de Riosucio y Aranzazu, 1,86 \% (5) de Neira y $10,04 \%$ de otros municipios.

Del total de pacientes, la mediana de creatinina fue $1,51 \mathrm{mg} / \mathrm{dl}(\mathrm{RIC}=1,22$ - 2,01). La mediana de TFGe por CKD-EPI fue de $42,7 \mathrm{mil} / \mathrm{min}(\mathrm{RIC}=30,6$ - 56,5) y la mediana de proteinuria fue de $246 \mathrm{mg}$ en 24 horas $(\mathrm{RIC}=100-1025)$. La mediana de glomérulos en la microscopia de luz fue de 18 (RIC=12 - 29); en la inmunofluorescencia, 3 (RIC $=2$ - 5); y en la microscopia electrónica, $2(\mathrm{RIC}=1$ - 2).

En la figura 1 se muestra el orden de frecuencia de los siete síndromes renales para indicación de biopsia renal en el total de pacientes y en los mayores de 65 años.

Los hallazgos histológicos más frecuentes fueron: nefropatía por inmunoglobulina A (NIgA) (20,9 \%, 56 pacientes), nefropatía hipertensiva $(19 \%, 51)$, glomeruloesclerosis focal segmentaria (GEFS) $(11,6 \%, 31)$, biopsia normal $(10,8 \%, 29)$, nefritis tubulointersticial (NTI) $(9,7 \%, 26)$, glomerulopatía diabética $(8,6 \%, 23)$, glomerulonefritis membranoproliferativa (GMNMP) $(3,7 \%, 10)$, glomerulonefritis proliferativa extracapilar (GNPE) $(3,4 \%, 9)$, glomerulonefritis crónica $(3 \%, 8)$, glomerulonefritis membranosa (GMNM) $(2,6 \%, 7)$, amiloidosis $(2,2 \%, 6)$, enfermedades hereditarias $(1,5 \%, 4)$, enfermedad de cambios mínimos (ECM) $(1,1 \%, 3)$; y otras causas $(1,8 \%, 5)$, entre las que se encuentran nefropatía por inmunoglobulina $M$, glomerulonefritis mesangioproliferativa y glomerulopatía fibrilar.

En los pacientes mayores de 65 años, los hallazgos más frecuentes fueron nefropatía hipertensiva (33 $\%, 34)$, NTI $(17,5 \%, 18)$, glomerulopatía diabética $(10,7 \%, 11), \operatorname{NIgA}(8,7 \%, 9)$, biopsia normal $(6,8$ $\%, 7)$, GEFS $(6,8 \%, 7)$, GNPE $(3,9 \%, 4)$, GMNM $(2,9 \%, 3)$, GMNMP $(2,9 \%, 3)$, glomerulonefritis crónica $(2,9 \%, 3)$, amiloidosis $(2,9 \%, 3)$ y otras causas $(0,97 \%, 1)$.

Los diagnósticos y sus principales formas de 


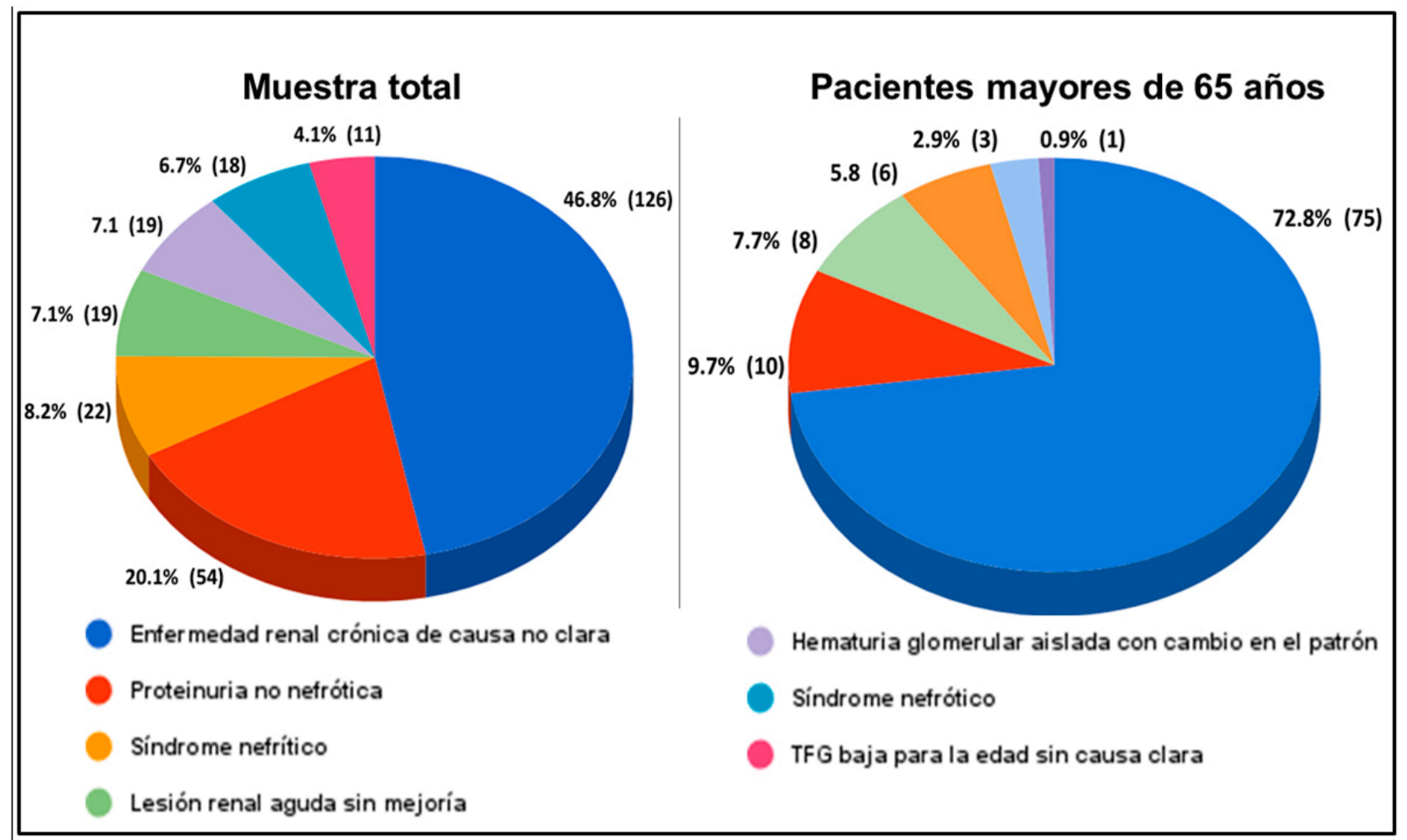

Figura 1. Frecuencia de síndromes renales/indicaciones de biopsia renal en la totalidad de pacientes y en pacientes mayores de 65 años

presentación, según el síndrome renal, se presentan en la muestra total y en mayores de 65 años, en las figuras 2 y 3 , respectivamente.

Del total de pacientes con NIgA, solo 5,3 \% (3) se consideraron por causa secundaria: 1 caso asociado a enfermedad autoinmune no especificada, 1 caso asociado a síndrome de Sjögren y 1 caso asociado a cirrosis hepática.

La nefropatía hipertensiva fue la principal causa de ERC sin causa clara $(35,7 \%)$ y en mayores de 65 años fue la primera causa de enfermedad renal. Entre las causas secundarias identificadas de GEFS se encontraron 8 misceláneas ( 5 casos por nefroesclerosis hipertensiva, y 3 por adaptaciones funcionales $\mathrm{y}$ estructurales), 4 hemodinámicas (obesidad), 2 por medicamentos, 1 inflamatoria (síndrome pulmón-riñón) y 1 por virus (VIH). De todos los casos de pacientes con síndrome nefrótico, la GEFS fue la principal causa $(38,8 \%, 7)$; y de todos los casos de proteinuria no nefrótica, fue la segunda causa $(24 \%, 13)$, después de la NIgA. La NTI fue la principal causa de LRA en el 29,3\% (5). En los pacientes mayores de 65 años, ocupó el segundo lugar de nefropatía. Las principales causas de NTI se describen en la tabla 1.

La glomerulopatía diabética fue la segunda causa en los pacientes con ERC sin causa clara (14,2 \%, 18).

Hubo 3 casos de GMNMP de etiología secundaria, 2 casos asociados a enfermedad autoinmune (crioglobulinemia y síndrome de Sjögren) y 1 caso asociado a proceso neoplásico. La GMNPE fue la principal causa de síndrome nefrítico $(27,2 \%)$. De los 9 casos, 7 tenían causa pauciinmune, 1 por inmunocomplejos y 1 sin dato. De la GMNM, 7 casos correspondieron a etiologías primarias y 1 a causa secundaria relacionada con autoinmunidad. De los 6 casos de amiloidosis, 4 correspondían a amiloidosis

110 Características clínicas e histológicas de las enfermedades parenquimatosas renales en una muestra de biopsias renales obtenidas entre el año 2002 y el 2017 en el departamento de Caldas, Colombia 
tipo AL (2 por mieloma múltiple) y dos casos a formas primarias (AA y otra no determinada). Los 4 casos de enfermedades hereditarias correspondieron a enfermedad por membrana basal delgada. En la categoría de otras enfermedades glomerulares, hubo 2 casos de nefropatía por IgM, 2 casos de GMN mesangioproliferativa y 1 caso de glomerulopatía fibrilar. En la tabla 2, se describen las medianas de creatinina, TFG y proteinuria en 24 horas para cada hallazgo histológico.

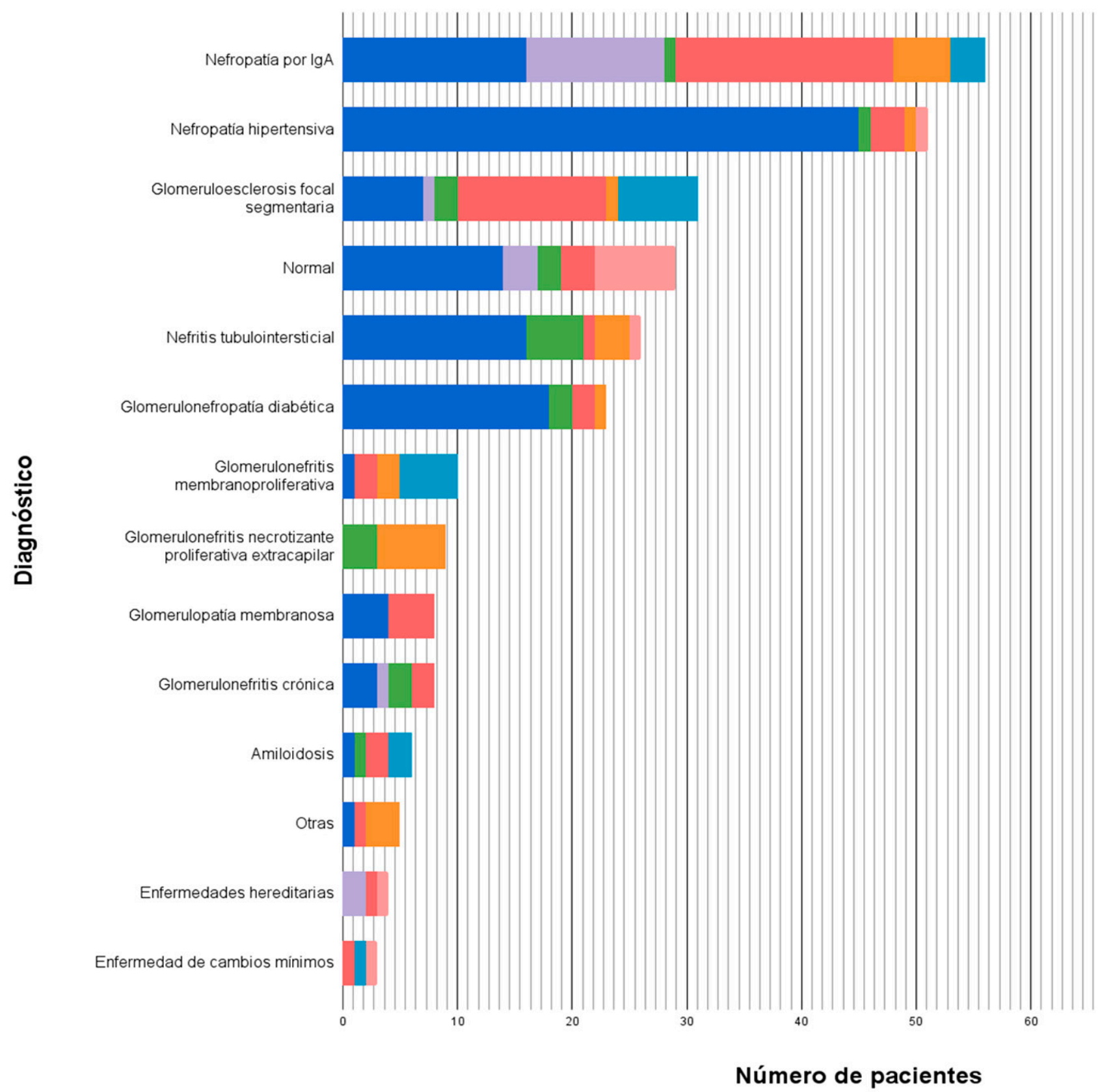

Figura 2. Síndrome renal según diagnóstico de enfermedad renal en todos los pacientes. 
Rev. Colomb. Nefrol. 2018;5(2): 107 - 117 http://www.revistanefrologia.org

http://dx.doi.org/10.22265/acnef.0.0.300

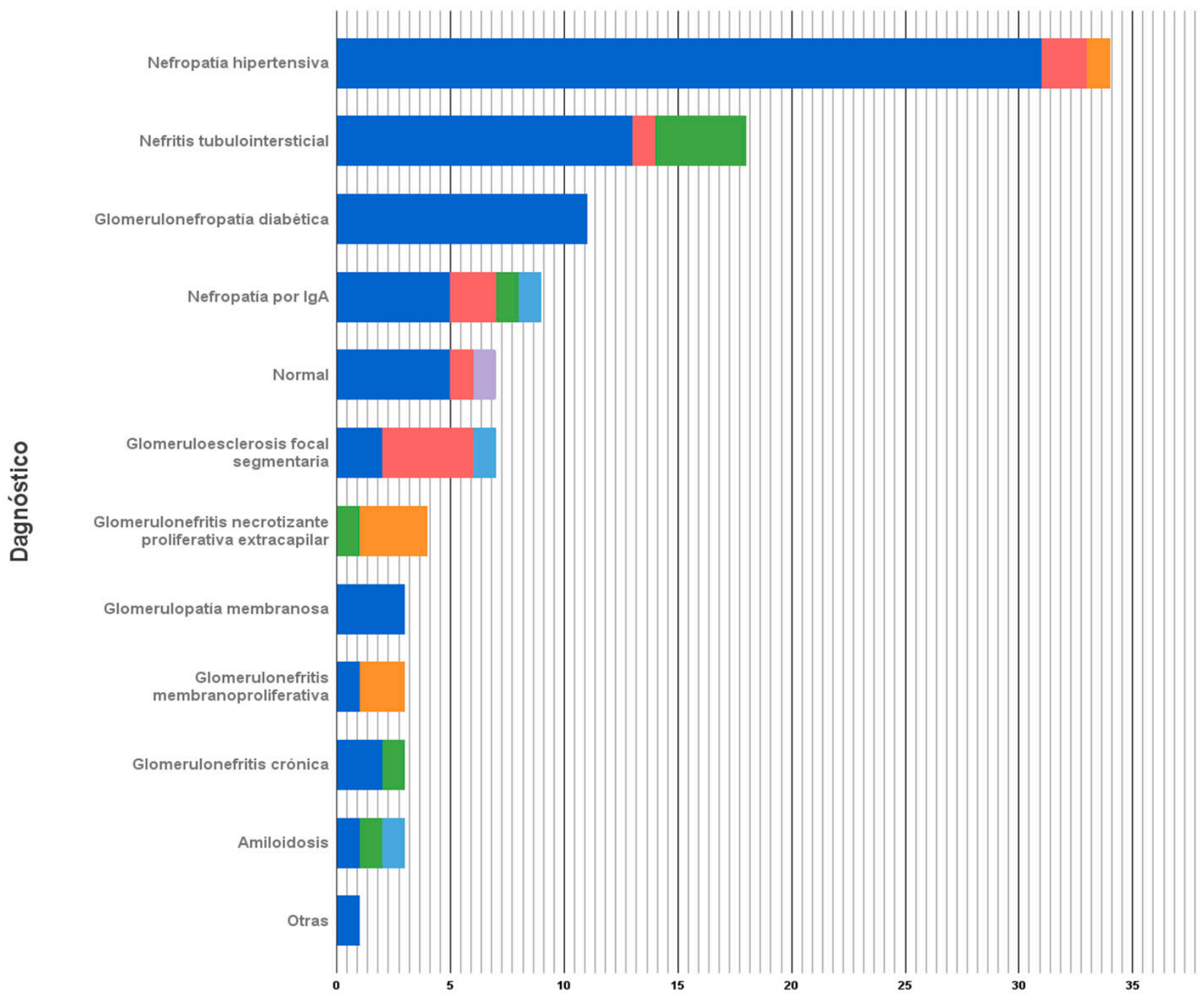

Número de casos

Figura 3. Síndrome renal según diagnóstico de enfermedad renal en pacientes mayores de 65 años.

Tabla 1. Causas de nefritis tubulointersticial.

\begin{tabular}{|l|c|}
\hline \multicolumn{1}{|c|}{ Causas de nefritis tubulointersticial } & Número de pacientes \\
\hline Analgésicos & 10 \\
\hline Enfermedad renal isquémica ateroesclerótica & 4 \\
\hline Infección urinaria & 3 \\
\hline Hiperuricemia & 2 \\
\hline Causa no clara & 2 \\
\hline Aminoglucósidos & 2 \\
\hline Uropatía obstructiva & 1 \\
\hline Síndrome de Sjögren & 1 \\
\hline Fenitoína & 1 \\
\hline Suma total & $\mathbf{2 6}$ \\
\hline
\end{tabular}

112 Características clínicas e histológicas de las enfermedades parenquimatosas renales en una muestra de biopsias renales obtenidas entre el año 2002 y el 2017 en el departamento de Caldas, Colombia 
Rev. Colomb. Nefrol. 2018;5(2): 107 - 117 http://www.revistanefrologia.org

http://dx.doi.org/10.22265/acnef.0.0.300

Tabla 2. Mediana de creatinina, TFGe CKD-EPI y

proteinuria según hallazgo histológico.

\begin{tabular}{|l|c|c|c|}
\multicolumn{1}{c|}{ Hallazgo histológico } & Mediana de creatinina (mg/dl) & $\begin{array}{c}\text { Mediana de TFGe CKD EPI } \\
(\mathrm{ml} / \mathrm{min})\end{array}$ & $\begin{array}{c}\text { Mediana de proteinuria (mg/24 } \\
\text { horas) }\end{array}$ \\
\hline Nefropatía hipertensiva & 1,63 & 38,3 & 150 \\
\hline Nefropatía por IgA & 1,39 & 52,6 & 324 \\
\hline Normal & 1,29 & 58,3 & 66 \\
\hline Nefritis tubulointersticial & 1,72 & 34,8 & 207 \\
\hline Glomerulonefropatía diabética & 1,6 & 34,6 & 688 \\
\hline $\begin{array}{l}\text { Glomeruloesclerosis focal } \\
\text { segmentaria }\end{array}$ & 1,5 & 41,3 & 3126 \\
\hline $\begin{array}{l}\text { Glomerulonefritis necrotizante } \\
\text { proliferativa extracapilar }\end{array}$ & 3,86 & 13 & 366 \\
\hline Glomerulonefritis crónica & 1,67 & 39,5 & 286 \\
\hline Glomerulopatía membranosa & 2,21 & 32,6 & 958 \\
\hline $\begin{array}{l}\text { Glomerulonefritis } \\
\text { membranoproliferativa }\end{array}$ & 3,79 & 14,2 & 1160 \\
\hline Amiloidosis & 3,38 & 16,5 & Sin valor \\
\hline $\begin{array}{l}\text { Enfermedad de cambios } \\
\text { mínimos }\end{array}$ & 1,14 & 53,7 & \\
\hline
\end{tabular}

\section{Discusión}

Los síndromes renales indican alteraciones en la integridad funcional y morfológica renal que se confirman en el diagnóstico histológico con la biopsia ${ }^{-}$. Además de identificar las lesiones potencialmente tratables $\mathrm{s}^{-}$, la biopsia renal aporta información para establecer la epidemiología de las enfermedades renales parenquimales ${ }^{3}$.

Aunque la NIgA ha sido la glomerulopatía más prevalente descrita a nivel mundial, la GMNM y la GEFS son las más comunes en otros países ${ }^{2}$. Esta variación depende de la etnia predominante en la población: en los asiáticos, la NIgA es más frecuente ${ }^{10}$; mientras que en los afroamericanos es la GEFS ${ }^{11}$.

En el estudio de Sim et al. ${ }^{9}$ en Norteamérica, la glomerulopatía más común fue la GEFS (38,9\%), seguida de la GMNM (12,7 \%). En la población afroamericana, la enfermedad más frecuente fue la GEFS (49,8\%). La distribución en blancos no hispánicos fue similar, pues prevaleció la GEFS
(35,9 \%), seguida de la GMNM (14,5\%) y la ECM $(11,9 \%)$. En los pacientes asiáticos, fue más frecuente la GEFS $(41,5 \%)$ y la $\operatorname{NIgA}(23,5 \%)$. Finalmente, en los hispánicos las más recurrentes fueron la GEFS (36 \%) y la GMNM (12,5\%).

En mayores de 60 años, Bomback et al. ${ }^{4}$ describen el síndrome nefrótico como la indicación más frecuente de biopsia, y la GMNM es el hallazgo más común $(32,1 \%)$. La amiloidosis es la segunda causa, seguida de la ECM. En pacientes muy ancianos o mayores de 80 años, los diagnósticos más frecuentes son variables en la literatura. Entre las causas descritas, están la GMNM, la glomerulonefritis pauciinmune y la nefroesclerosis benigna ${ }^{12}$.

En Argentina, Arenas y et al. $\frac{13}{r}$ reportaron como principales indicaciones de biopsia la insuficiencia renal con alteraciones del sedimento urinario (azoemia más proteinuria y hematuria), seguida por síndrome nefrótico y proteinuria aislada. Los principales diagnósticos fueron GMN por lupus y GMN proliferativa mesangial sin depósito de IgA. 
En Lima, Perú, Hurtado et al. ${ }^{14}$ describieron como enfermedad glomerular más común la secundaria a LES, seguida de GMNMP y GEFS. La NIgA fue poco frecuente. En Brasil, Cruz et al. $\frac{15}{}$ reportaron que la GEFS fue el hallazgo más frecuente, seguido de GMNM. La NIgA, ECM y GMNMP tuvieron una frecuencia baja.

En Colombia, el estudio de Mejía et al. $\frac{16}{}$ en Medellín, Antioquia, tiene la mayor muestra recolectada a la fecha, con 383 biopsias. Las indicaciones de biopsia renal más frecuentes fueron síndrome nefrótico (42\%), glomerulonefritis $(28,2$ \%)y hematuria aislada (19,8\%). Las glomerulopatías más frecuentes fueron ECM (30\%), GEFS (21,1 $\%)$ y glomerulonefritis proliferativa endocapilar difusa $(10,4 \%)$. La principal patología secundaria fue el lupus. La GEFS se presentó principalmente como síndrome nefrótico (69,2\%), así como en la GMNM (74,3 \%). Sin embargo, en este reporte solo en algunos casos se sometió la muestra a estudio por inmunofluorescencia (IF), y ninguno a microscopia electrónica (ME).

El estudio de Gómez-Jiménez et al. $\frac{17}{}$, realizado en Medellín, Antioquia, reporta las biopsias de 11 pacientes gestantes. Hubo 4 casos de nefritis lúpica, 2 pacientes con GNRP por GN extracapilar y poliangiítis granulomatosa y 3 con síndrome nefrótico por GEFS.

El estudio de Serna-Flórez et al. $\frac{18}{}$ en Armenia, Quindío, incluyó 168 pacientes. Las glomerulopatías primarias principales fueron GEFS (17,58 \%), $\operatorname{NIgA}(17,58 \%)$, GMNM (14,29 \%) y enfermedad de cambios mínimos $(13,19 \%)$. En mayores de 60 años, la principal fue GEFS (37,5\%). Las glomerulopatías secundarias $(14,95 \%)$ fueron representadas principalmente por la nefropatía lúpica $(81,25 \%)$. No se especifica en el estudio si el total de las biopsias fueron sometidas a estudio por IF y ME.

El estudio de Coronado et al. $\frac{19}{}$ en Ibagué, Tolima, incluyó 181 pacientes. Las indicaciones para biopsia, en su orden, fueron síndrome nefrótico $(35,51 \%)$, seguido de insuficiencia renal $(32,7 \%)$. Todas las biopsias se analizaron por inmunohistoquímica, y 97,24\% por microscopia electrónica. Los diagnósticos de glomerulopatía fueron, en su orden, GMN lúpica (27,6\%), GMNM $(18,2 \%)$, GEFS (14,9\%), nefropatía crónica (6\%), $\operatorname{NIgA}(5,5 \%)$ y $\operatorname{ECM}(4,9 \%)$.

Nuestro estudio es el quinto en Colombia en describir los hallazgos de una serie de biopsias renales. A diferencia del reporte de Mejía et al. $\frac{16}{}$, solo se seleccionaron muestras que fueron sometidas a estudios con microscopia de luz, IF y ME. Este trabajo también difiere del estudio de Coronado et al. $\frac{19}{}$, en el que excluyó pacientes con nefritis lúpica. Es el primero que diferencia enfermedades renales primarias de secundarias y las reporta en forma aislada para el grupo de pacientes mayores de 65 años.

El promedio de edad fue mayor, comparado con el reportado en otros estudios nacionales $\frac{16,17,18,19}{} \mathrm{e}$ internacionales. $\frac{13,14,15}{}$. La mediana de creatinina fue mayor y la TFG menor, comparada con los estudios colombianos de Serna-Flórez et al. $\stackrel{11}{ }$ y Coronado et al. $\stackrel{12}{\text {. }}$

En este estudio, la ERC sin causa clara fue la principal indicación de biopsia. La edad avanzada de la población y la no inclusión de pacientes con nefropatía lúpica explicarían este hallazgo. La proteinuria no nefrótica ocupó el segundo lugar como indicación de biopsia y se mantuvo, aunque en menor proporción, en los mayores de 65 años. El síndrome nefrótico ocupó un papel menos frecuente (sexta causa en la población general y quinta en mayores de 65 años).

El síndrome nefrítico ocupó el tercer lugar como forma de presentación clínica. Al contrario, la LRA es la tercera causa en mayores de 65 años $(7,8 \%)$. La hematuria glomerular con cambio en el patrón en mayores de 65 años solo tuvo 1 caso.

En esta serie de casos, la NIgA fue el hallazgo histológico más frecuente, de manera similara la mayoría de poblaciones en el mundo ${ }^{9,20}$, pero diferente a los estudios previos latinoamericanos ${ }^{13-19}$. Habitualmente, la presentación clínica más frecuente en jóvenes es la hematuria, y el sedimento anormal en ancianos ${ }^{21}$.

114 Características clínicas e histológicas de las enfermedades parenquimatosas renales en una muestra de biopsias renales obtenidas entre el año 2002 y el 2017 en el departamento de Caldas, Colombia 


\section{http://dx.doi.org/10.22265/acnef.0.0.300}

En este estudio, la proteinuria no nefrótica, ERC sin causa clara en similar proporción y la hematuria glomerular con cambio en el patrón fueron las principales formas de presentación clínica.

El diagnóstico de la nefroesclerosis hipertensiva aumenta con la edad $\underline{22}$. Sin embargo, usualmente se hace el diagnóstico clínicamente por el rechazo para realizar biopsia renal en esta población ${ }^{23}$. En este estudio, fue el segundo hallazgo histológico en toda la muestra y el principal en mayores de 65 años.

En los adultos con proteinuria sometidos a biopsia renal, la GEFS representa el $35 \%$ de los $\operatorname{casos}^{24}$. En este estudio, en los pacientes con síndrome nefrótico, la GEFS fue el principal hallazgo $(38,8$ $\%$ ) y la proteinuria no nefrótica se presentó en un 24 $\%$ de los casos. A diferencia de los estudios previos en Colombia $\frac{16-19}{y}$ y los de latinoamérica ${ }^{13-15}$ que reportan frecuencias de hasta el $5 \%$, en este estudio el 9,7\% fueron hallazgos histológicos. En mayores de 65 años fue más frecuente $(17,5 \%)$.

Como se describe en la literatura ${ }^{25}$, la principal causa de NTI en este estudio fueron los analgésicos asociados al manejo de enfermedades articulares $(38,8 \%)$. La enfermedad renal isquémica $\mathrm{y}$ ateroesclerótica más común en personas de edad avanzada ocupó el segundo lugar (15,3\%).

En la diabetes mellitus, la biopsia no se indica rutinariamente en todos los casos, en especial en pacientes con historia y progresión típica de la enfermedad (progresión en la disminución de la TFG y albuminuria persistente). La indicación se realiza en caso de sospecha de otra enfermedad renal o si hay características atípicas presentes $\frac{26,27}{}$. El grupo de pacientes de este estudio representa la presentación atípica que se manifiesta principalmente como ERC sin causa clara y como única manifestación en los mayores de 65 años.

Este estudio es el segundo con mayor muestra en Colombia, las definiciones de los síndromes renales son similares a las de los estudios previos y solo se incluyeron biopsias con los tres estudios (microscopia de luz, inmunofluorescencia y microscopia electrónica).
Sin embargo, no se pueden hacer asociaciones, ya que se trata de un estudio descriptivo tipo serie de casos. Por lo tanto, se requieren estudios posteriores en Colombia. Además, no se incluyeron pacientes con nefropatía lúpica que tiene un papel importante como causa de nefropatía secundaria. Finalmente, el tipo de estudio aumenta el riesgo de sesgos y, al ser una muestra por conveniencia, no se puede generalizar a la población caldense y, por lo tanto, a la colombiana.

Se requieren estudios que permitan evaluar asociaciones entre las indicaciones renales y las diferentes glomerulopatías. Los resultados actuales y los de los estudios previos a nivel nacional son la herramienta disponible para tener una probabilidad pretest ante un paciente con un síndrome renal y la sospecha de enfermedad renal parenquimatosa.

\section{Conflicto de intereses}

Los autores declaran no tener conflicto de interés actual o potencial.

\section{Responsabilidades éticas \\ Protección de personas y animales}

Los autores declaran que para esta investigación no se han realizado experimentos en seres humanos ni en animales.

\section{Confidencialidad de los datos}

Los autores declaran que han seguido los protocolos de su centro de trabajo sobre la publicación de datos de pacientes.

\section{Derecho a la privacidad y consentimiento informado}

Los autores declaran que en este artículo no aparecen datos de pacientes.

\section{Contribución aporte de los autores}

Se declara que los autores han participado en el diseño, realización e interpretación de los resultados. 


\section{Referencias}

1. Restrepo CA, Buitrago CA, Torres J, Serna J. Nefrología básica 2. Manizales: Editorial La Patria; 2012.

2. Dhaun N, Bellamy CO, Cattran D, Kluth DC. Utility of renal biopsy in the clinical management of renal disease. Kidney Int. 2014;85(5):1039-1048. https://doi.org/10.1038/ki.2013.512

3. Ismail MI, Lakouz K, Abdelbary E. Clinicopathological correlations of renal pathology: A single center experience. Saudi J Kidney Dis Traspl. 2016;27(3):557-562. https://doi.org/10.4103/1319-2442.182399.

4. Bomback AS, Herlitz LC, Markowitz GS. Renal biopsy in the elderly and very elderly: useful or not?. Adv Chronic Kidney Dis. 2012;19(2):61-67. https://doi.org/10.1053/j.ackd.2011.09.003

5. Carrilho-Mota P. Indicaçoes actuáis para biopsia renal. Acta Med Port. 2005;18(2):147-151.

6. Imitiaz S, Nasir K, Drohlia MF, Salman B, Ahmad A. Frecuency of kidney diseases and clinical indications of pediatric renal biopsy: A single center experience. Indian J Nephrol. 2016;26(3):199-205. https://doi.org/10.4103/0971-4065.159304

7. Matsushita K, Mahmoodi B, Woodward M, Emberson JR, Jafar TH, Jee SH, et al. Comparison of risk prediction using the CKDEPI equation and the MDRD study equation for estimated glomerular filtration rate. JAMA. 2012;307(18):1941-1951. https://doi. org/10.1001/jama.2012.3954

8. Papadakis MA, Mcphee S. Current Medical Diagnosis \& Treatment 2017. New York: McGraw Hill; 2017.

9. Sim JJ, Batech M, Hever A, Harrison TN, Avelar T, Kanter MH, et al. Distribution of biopsy proven presumed primary glomerulonephropathies in 2000-2011 among a racially and ethnically diverse US population. Am J Kidney Dis. 2016;68(4):533-544. https://doi.org/10.1053/j.ajkd.2016.03.416

10. Woo KT, Chan CM, Chin YM, Choong HL, Tan HK, Foo M, et al. Global evolutionary trend of the prevalence of primary glomerulonephritis over the past three decades. Nephron Clin Pract. 2010;116(4):c337-c346. https://doi.org/10.1159/000319594

11. Korbet SM, Genchi RM, Borok RZ, Schwartz MM. The racial prevalence of glomerular lesions in nephrotic adults. Am J Kidney Dis. 1996;27(5):647-651.

12. Nair R, Bell JM, Walker PD. Renal biopsy in patients aged 80 years and older. Am J Kidney Dis. 2004;44(4):618-626.

13. Arenas PG, Diller A, Orias M, Arteaga J, Douchat W, Massari P. Biopsias renales: frecuencia, indicaciones y resultados actuales en un centro hospitalario. Nefrología Argentina. 2005;3(2):55-65.

14. Hurtado A, Escudero E, Stronquist CS, Urcia J, Hurtado ME, Gretch D, et al. Distinct patterns of glomerular disease in Lima, Perú. Clin Nephrol. 2000;53:325-332.

15. Cruz HM, Penna D de O, Saldanha LB, Cruz J, Luiz P, Marcondes M, et al. Histopathologic study of primary glomerulopathies: retrospective analysis of 197 renal biopsies (1985-1987). Rev Hosp Clin Fac Med Sao Paulo. 1989;44(3):94-99.

16. Mejia G, Builes M, Arbelaez M, Henao JE, Arango JL, Garcia A. Descripción clinicopatológica de las enfermedades glomerulares. Acta Med Colomb. 1989;14(6):369-374.

17. Gómez-Jiménez JM, Arias LF. Enfermedades glomerulares durante la gestación. Serie de casos y revisión de la literatura. Revista Colombiana de obstetricia y Ginecología. 2008;59(4):343-348.

18. Serna-Florez J, Torres-Saltarín J, Serrano-Mass D. Enfermedades renales diagnósticadas por biopsia: descripción clínica, histológica y epidemiológica. Resultados de la población atendida entre 1992 y 2010 en el servicio de nefrología del Hospital Universitario San Juan de Dios. Armenia (Colombia). Méd UIS.2011;24(1):39-43.

19. Coronado CY, Echeverry I. Descripción clínicopatológica de las enfermedades glomerulares. Acta MedColomb. 2016:41(2):125129.

20. McGrogan A, Franssen CF, de Vries CS. The incidence of primary glomerulonephritis worldwide: a systematic review of the literature. Nephrol Dial Transplant. 2011;26(2):414-430. https://doi.org/10.1093/ndt/gfq665

21. Rodrigues JC, Haas M, Reich HN. IgA Nephropathy. Clin J Am Soc Nephrol. 2017;12(4):677-686.

22. Freedman BI, Iskander SS, Buckalew VM Jr, Burkart JM, Appel RG. Renal biopsy findings in presumed hypertensive nephrosclerosis. Am J Nephrol. 1994;14(2):90-94. https://doi.org/10.1159/000168695

116 Características clínicas e histológicas de las enfermedades parenquimatosas renales en una muestra de biopsias renales obtenidas entre el año 2002 y el 2017 en el departamento de Caldas, Colombia

e2500-5006 Revista Colombiana de Nefrología 
Rev. Colomb. Nefrol. 2018;5(2): 107 - 117 http://www.revistanefrologia.org

http://dx.doi.org/10.22265/acnef.0.0.300

23. Moutzouris DA, Herlitz L, Appel GB, Markowitz GS, Freudenthal B, Radhakrishnan J, et al. Renal biopsy in the very elderly. Clin J Am Soc Nephrol. 2009;4:1073-1082. https://doi.org/10.2215/CJN.00990209

24. Kitiyakara C, Eggers P, Kopp JB. Twenty-one-year trend in ESRD due to focal segmental glomerulosclerosis in the United States. Am J Kidney Dis. 2004;44(5):815-825.

25. De Broe ME, Elseviers MM. Over-the-counter analgesic use. J Am Soc Nephrol. 2009;20(10):2098-2103. https://doi.org/10.1681/ ASN.2008101097

26. Tang SC, Chan GC, Lai KN. Recent advances in managing and understanding diabetic nephropathy. F1000Res. 2016;5. https:// doi.org/10.12688/f1000research.7693.1

27. Aristizábal Gómez LY, Restrepo Valencia CA., Aguirre Arango JV. Clinical characteristic of a population of diabetics type 2 with alteration in the renal function non macroalbuminuric. Rev Colomb Nefrol. 2017;4(2):149-158. https://doi.org/10.22265/ acnef.4.2.271 